Received by the editors: November 25, 2019; Accepted: May 16, 2020

\title{
A SUBCLASS OF PSEUDO-TYPE MEROMORPHIC BI-UNIVALENT FUNCTIONS
}

\author{
Adnan Ghazy ALAMOUSH \\ Faculty of Science, Taibah University, SAUDI ARABIA
}

\begin{abstract}
In this paper, a new subclass of pseudo-type meromorphic biunivalent functions is defined on $\triangle=\{z \mid: z \in C$ and $1<|z|<\infty\}$, we derive estimates on the initial coefficient $\left|b_{0}\right|,\left|b_{1}\right|$ and $\left|b_{2}\right|$. Relevant connections of the new results with various well-known results are indicated.
\end{abstract}

\section{Introduction}

Let $A$ denote the class of functions $f(z)$ of the form:

$$
f(z)=z+\sum_{n=2}^{\infty} a_{n} z^{n}
$$

which are analytic in the open unit open disk $U=\{z: z \in C,|z|<1\}$. Also, let the class of univalent and normalized analytic function in the unit disc $U$ be denoted by $S$ with the normalization conditions

$$
f(0)=0=f^{\prime}(0)-1 .
$$

Furthermore, bi-univalency concept is extended to the class of meromorphic functions defined on $\triangle=\{z: z \in C, 1<|z|<\infty\}$. For this aim, let $\Sigma$ denote the class of meromorphic univalent functions $g$ of the form

$$
g(z)=z+\sum_{n=0}^{\infty} \frac{b_{n}}{z^{n}}
$$

defined on the domain $\triangle$. It is well known that every function $g \in \Sigma$ has an inverse $g^{-1}=h$, defined by

2020 Mathematics Subject Classification. Primary 11B39, 30C45, 33C45; Secondary 30C50, $33 \mathrm{C} 05$.

Keywords and phrases. Analytic functions, univalent functions, meromorphic functions, biunivalent functions, coefficient bounds, pseudo functions.

\agalamoush@taibahu.edu.sa

(D) 0000-0003-3687-9195.

(C)2020 Ankara University

Communications Faculty of Sciences University of Ankara-Series A1 Mathematics and Statistics 
and

$$
g^{-1}(g(z))=z \quad(z \in \triangle)
$$

$g^{-1}(g(w))=w \quad(M<|w|<\infty, M>0)$,

where

$g^{-1}(w)=h(w)=w+\sum_{n=0}^{\infty} \frac{B_{n}}{w^{n}}=w-b_{0}-\frac{b_{1}}{w}-\frac{b_{1} b_{0}+b_{2}}{w^{2}}-\frac{b_{1}^{2}+b_{1} b_{0}^{2}+2 b_{0} b_{2}+b_{3}}{w^{3}}+\ldots$.

A simple computation shows that

$$
\begin{gathered}
w=g(h(w))=\left(b_{0}+B_{0}\right)+w+\frac{b_{1}+B_{1}}{w}+\frac{B_{2}-b_{1} B_{0}+b_{2}}{w^{2}} \\
+\frac{B_{3}-b_{1} B_{1}+b_{1} B_{0}^{2}-2 b_{2} B_{0}+b_{3}}{w^{3}}+\ldots
\end{gathered}
$$

Comparing the initial coefficients in (4), we find that

$$
\begin{aligned}
b_{0}+B_{0}=0 & \Rightarrow B_{0}=-b_{0} \\
b_{1}+B_{1}=0 & \Rightarrow B_{1}=-b_{1} \\
B_{2}-b_{1} B_{0}+b_{2}=0 & \Rightarrow B_{2}=-\left(b_{2}+b_{1} b_{0}\right) \\
B_{3}-b_{1} B_{1}+b_{1} B_{0}^{2}-2 b_{2} B_{0}+b_{3}=0 & \Rightarrow B_{3}=-\left(b_{3}+2 b_{0} b_{1}+b_{1} b_{0}^{2}+b_{1}^{2}\right) .
\end{aligned}
$$

A function $f \in \Sigma$ is said to be meromorphic bi-univalent if $f^{-1} \in \Sigma$. The family of all meromorphic bi-univalent functions is denoted by $\Sigma^{\prime}$. Estimates on the coefficient of meromorphic univalent functions were investigated by some researchers recently; for example, Schiffer 11 obtained the estimate $\left|b_{2}\right|<\frac{3}{2}$ for meromorphic univalent functions $f \in S$ with $b_{0}=0$. Also, Duren 12 obtained the inequality $\left|b_{2}\right|<\frac{2}{n+1}$ for $f \in S$ with $b_{k}=0,1 \leq k \leq \frac{n}{2}$. Springer 8 used variational methods to prove that proved that

$$
\left|B_{3}\right|<1 \text { and }\left|B_{3}+\frac{1}{2} B_{1}^{2}\right|<\frac{1}{2}
$$

and conjectured that

$$
\left|B_{2 n-1}\right| \leq \frac{(2 n-2) !}{n !(n-1) !}(n=1,2, \ldots) .
$$

Later on, Kubota 16 has proved that the Springer conjecture is true for $\mathrm{n}=3 ; 4 ; 5$. Furthermore Schober 7 obtained sharp bounds for $\left|B_{2 n-1}\right|$ if $1 \leq n \leq 7$. Recently. Kapoor and Mishra [5] found the coefficient estimates for a class consisting of inverses of meromorphic starlike univalent functions of order $\alpha$ in $U^{*}$.

Recently, some several researchers such as ( see 1], 2], 3], [4, 6], 9], 13] 14]) introduced new subclasses of meromorphically bi-univalent functions and obtained estimates on the initial coefficients for functions belonging to these subclasses. 
In 2013, Babalola 10 defined a new subclass $\lambda$-pseudo starlike function of order $0 \leq \beta<1$ satisfying the analytic condition

$$
\Re\left\{\frac{z\left(f(z)^{\prime \lambda}\right.}{f(z)}\right\}>\beta(\lambda \geq 1, z \in U) .
$$

In particular, Babalola [10] proved that all $\lambda$-pseudo-starlike functions are Bazilevic of type $1-\frac{1}{\lambda}$ and order $\beta^{\frac{1}{\lambda}}$ and are univalent in open unit disk $U$.

Motivated by the earlier work of $([9],[15])$, in the present paper, we introduce a new subclasses of the class $\Sigma^{\prime}$ and the estimates for the coefficients $\left|b_{0}\right|,\left|b_{1}\right|$ and $\left|b_{2}\right|$ are investigated. Some new consequences of the new results are also pointed out.

\section{Coefficient Bounds for the Function Class $\Sigma_{h, p}^{\prime}(\lambda, \mu)$}

We begin by introducing the function class $\Sigma_{h, p}^{\prime}(\lambda, \mu)$ by means of the following definition.

Definition 2.1. Let the functions $h ; p: \triangle \rightarrow C$ be analytic functions and

$$
h(z)=1+\frac{h_{1}}{z}+\frac{h_{2}}{z^{2}}+\frac{h_{3}}{z^{3}}+\cdots, \quad p(z)=1+\frac{p_{1}}{z}+\frac{p_{2}}{z^{2}}+\frac{p_{3}}{z^{3}}+\cdots,
$$

such that

$$
\min \{\Re(h(z)), \Re(p(z))\}>0, z \in \triangle .
$$

A function $g(z) \in \Sigma^{\prime}$ given by (2) is said to be in the class $\Sigma_{h, p}^{\prime}(\lambda, \mu)$ if the following conditions are satisfied:

$$
\begin{gathered}
g \in \Sigma^{\prime} \text { and } 1+\frac{1}{\gamma}\left[(1-\lambda)\left(\frac{g(z)}{z}\right)^{\mu}+\lambda\left(\frac{z\left(g(z)^{\prime \mu}\right.}{g(z)}\right)-1\right] \in h(\triangle), \\
(0<\lambda \leq 1, \mu \geq 1, z \in \triangle)
\end{gathered}
$$

and

$$
\begin{gathered}
1+\frac{1}{\gamma}\left[(1-\lambda)\left(\frac{h(w)}{w}\right)^{\mu}+\lambda\left(\frac{w\left(h(w)^{\prime \mu}\right.}{h(w)}\right)-1 \in p(\triangle)\right] \\
(0<\lambda \leq 1, \mu \geq 1, w \in \triangle),
\end{gathered}
$$

where $g \in \Sigma^{\prime}$ and $\gamma \in C \backslash\{0\}$ and the function $h$ is given by (3).

Remark 2.1. There are many choices of $h$ and $p$ which would provide interesting subclasses of class $\Sigma_{h, p}^{\prime}(\lambda, \mu)$.

(1) If we take

$$
h(z)=p(z)=\left(\frac{1+\frac{1}{z}}{1-\frac{1}{z}}\right)^{\alpha}=1+\frac{2 \alpha}{z}+\frac{2 \alpha^{2}}{z^{2}}+\cdots,(0<\alpha \leq 1, z \in \triangle) .
$$


So it is easy to verify that the functions $h(z)$ and $p(z)$ satisfy the hypotheses of Definition 2.1. If $f \in \Sigma_{\alpha}^{\prime}(\lambda, \mu)$. Then

$$
\begin{gathered}
\left|\arg \left(1+\frac{1}{\gamma}\left[(1-\lambda)\left(\frac{g(z)}{z}\right)^{\mu}+\lambda\left(\frac{z\left(g(z)^{\prime \mu}\right.}{g(z)}\right)-1\right]\right)\right|<\frac{\alpha \pi}{2} \\
(0<\lambda \leq 1,0<\alpha \leq 1, \mu \geq 1, z \in \triangle),
\end{gathered}
$$

and

$$
\left|\arg \left(1+\frac{1}{\gamma}\left[(1-\lambda)\left(\frac{h(w)}{w}\right)^{\mu}+\beta\left(\frac{w\left(h(w)^{\prime \mu}\right.}{h(w)}\right)-1\right]\right)\right|<\frac{\alpha \pi}{2}
$$$$
(0<\lambda \leq 1,0<\alpha \leq 1, \mu \geq 1, w \in \triangle) \text {, }
$$

where $g(z) \in \Sigma^{\prime}$ and $\gamma \in C \backslash\{0\}$ and the function $h$ is given by (3).

(2) If we take

$$
h(z)=p(z)=\frac{1+\frac{1-2 \beta}{z}}{1-\frac{1}{z}}=1+\frac{2(1-\beta)}{z}+\frac{2(1-\beta)}{z^{2}},(0 \leq \beta<1, z \in \triangle) .
$$

So it is easy to verify that the functions $h(z)$ and $p(z)$ satisfy the hypotheses of Definition 2.1. If $f \in \Sigma_{\beta}^{\prime}(\lambda, \mu)$. Then

$$
\begin{gathered}
\Re\left(1+\frac{1}{\gamma}\left[(1-\lambda)\left(\frac{g(z)}{z}\right)^{\mu}+\lambda\left(\frac{z\left(g(z)^{\prime \mu}\right.}{g(z)}\right)-1\right]\right)>\beta \\
(0<\lambda \leq 1,0 \leq \beta<1, \mu \geq 1, z \in \triangle),
\end{gathered}
$$

and

$$
\Re\left(1+\frac{1}{\gamma}\left[(1-\lambda)\left(\frac{h(w)}{w}\right)^{\mu}+\beta\left(\frac{w\left(h(w)^{\prime \mu}\right.}{h(w)}\right)-1\right]\right)>\beta
$$

$(0<\lambda \leq 1,0 \leq \beta<1, \mu \geq 1, w \in \triangle)$,

where $g \in \Sigma^{\prime}$ and $\gamma \in C \backslash\{0\}$ and the function $h$ is given by (3).

Theorem 2.1. Let $g(z)$ be given by (2) be in the class $\Sigma_{\alpha}^{\prime}(\lambda, \mu)$. Then

$$
\left|b_{0}\right| \leq \min \left\{\sqrt{\frac{|\gamma|^{2}\left(\left|h_{1}\right|^{2}+\left|p_{1}\right|^{2}\right)}{2(\mu-\lambda \mu-\lambda)^{2}}}, \sqrt{\frac{|\gamma|\left(\left|h_{2}\right|+\left|p_{2}\right|\right)}{|\mu(\mu-1)(1-\lambda)+2 \lambda|}}\right\}
$$

and

$$
\begin{gathered}
\left|b_{1}\right| \leq \min \left\{\frac{|\gamma|\left(\left|h_{2}\right|+\left|p_{2}\right|\right)}{|2(\mu(\mu-1)(1-\lambda)+2 \lambda)|},\right. \\
\left.\frac{|\gamma|}{|(\mu-\lambda-2 \lambda \mu)|}\left(\sqrt{\frac{\left|h_{2}\right|^{2}+\left|p_{2}\right|^{2}}{2}+\frac{[\mu(\mu-1)(1-\lambda)+2 \lambda]^{2}\left[h_{1}^{2}+p_{1}^{2}\right]^{2}}{16(\mu-\lambda \mu-\lambda)^{2}}}\right)\right\},
\end{gathered}
$$

and

$$
\left|b_{2}\right| \leq \frac{|\gamma|}{2|(\mu-\lambda-3 \lambda \mu)|}\left[\frac{(\mu(\mu-1)(\mu-2)(1-\lambda)-6 \lambda) \gamma^{2}\left|p_{1}\right|^{3}}{3\left|(\mu-\lambda \mu-\lambda)^{3}\right|}\right.
$$




$$
\begin{aligned}
& +\frac{2 \mu(\mu-1)(1-\lambda)+8 \mu \lambda-2 \mu+6 \lambda}{2 \mu(\mu-1)(1-\lambda)-(1-\lambda) \mu+5 \lambda+4 \lambda \mu}\left|h_{3}\right| \\
& \left.+\frac{2 \mu(\mu-1)(1-\lambda)+2 \mu \lambda+4 \lambda}{2 \mu(\mu-1)(1-\lambda)-(1-\lambda) \mu+5 \lambda+4 \lambda \mu}\left|p_{3}\right|\right]
\end{aligned}
$$

Proof. Let $g \in \Sigma_{\alpha}^{\prime}(\lambda, \mu)$. Then, by Definition 2.1 of meromorphically bi-univalent function class $\Sigma_{\alpha}^{\prime}(\lambda, \mu)$, the conditions (6) and (7) can be rewritten as follows:

$$
1+\frac{1}{\gamma}\left[(1-\lambda)\left(\frac{g(z)}{z}\right)^{\mu}+\lambda\left(\frac{z\left(g(z)^{\prime \mu}\right.}{g(z)}\right)-1\right]=h(z) \quad(z \in \triangle)
$$

and

$$
1+\frac{1}{\gamma}\left[(1-\lambda)\left(\frac{h(w)}{w}\right)^{\mu}+\beta\left(\frac{w\left(h(w)^{\prime \mu}\right.}{h(w)}\right)-1\right]=p(w), \quad(w \in \triangle)
$$

respectively. Here, and in what follows, the functions $h(z) \in P$ and $p(w) \in P$ have the following forms:

$$
h(z)=1+\frac{p_{1}}{z}+\frac{p_{2}}{z^{2}}+\frac{p_{3}}{z^{3}}+\cdots \quad(z \in \triangle)
$$

and

$$
p(w)=1+\frac{q_{1}}{w}+\frac{q_{2}}{w^{2}}+\frac{q_{3}}{w^{3}}+\cdots \quad(w \in \triangle)
$$

upon substituting from (13) and (14) into (11) and (12), respectively, and equating the coefficients, we get

$$
\begin{gathered}
\frac{(\mu-\lambda \mu-\lambda)}{\gamma} b_{0}=h_{1} \\
\frac{1}{2 \gamma}\left[(\mu(\mu-1)(1-\lambda)+2 \lambda) b_{0}^{2}+2(\mu-\lambda-2 \lambda \mu) b_{1}\right]=h_{2} \\
\frac{1}{6 \gamma}[\mu(\mu-1)(\mu-2)(1-\lambda)-\lambda] b_{0}^{3}+\frac{1}{\gamma}[\mu(\mu-1)(1-\lambda)+2 \lambda+\lambda \mu] b_{0} b_{1} \\
+\frac{1}{\gamma}[\mu-\lambda-3 \mu \lambda] b_{2}=h_{3} \\
-\frac{(\mu-\lambda \mu-\lambda)}{\gamma} b_{0}=p_{1} \\
\frac{1}{2 \gamma}\left[(\mu(\mu-1)(1-\lambda)+2 \lambda) b_{0}^{2}+2(\lambda-\mu+2 \lambda \mu) b_{1}\right]=p_{2}
\end{gathered}
$$

and

$$
\begin{aligned}
& \frac{1}{6 \gamma}\left[6 \lambda-(\mu(\mu-1)(\mu-2)(1-\lambda)) b_{0}^{3}+6(\mu(\mu-1)(1-\lambda)\right. \\
& \left.-\mu(1-\lambda)+3 \lambda+3 \lambda \mu) b_{0} b_{1}+6(\lambda-\mu+3 \mu \lambda) b_{2}\right]=p_{3} .
\end{aligned}
$$

From (15) and (18), we find that

$$
h_{1}=-q_{1}
$$

and

$$
2(\mu-\lambda \mu-\lambda)^{2} b_{0}^{2}=\gamma^{2}\left(h_{1}^{2}+p_{1}^{2}\right)
$$


that is,

$$
\left|b_{0}\right|^{2} \leq \frac{|\gamma|^{2}\left(\left|h_{1}\right|^{2}+\left|p_{1}\right|^{2}\right)}{2(\mu-\lambda \mu-\lambda)^{2}} .
$$

Adding (16) and (19), we get

$$
[(\mu(\mu-1)(1-\lambda)+2 \lambda)] b_{0}^{2}=\gamma\left(h_{2}+p_{2}\right)
$$

that is,

$$
\left|b_{0}\right|^{2} \leq \frac{|\gamma|\left(\left|h_{2}\right|+\left|p_{2}\right|\right)}{|\mu(\mu-1)(1-\lambda)+2 \lambda|}
$$

From (23) and (25) we get the desired estimate on the coefficient $\left|b_{0}\right|$ as asserted in $(8)$.

Next, in order to find the bound on $\left|b_{0}\right|$, by subtracting the equation (16) from the equation (19), we get

$$
2(\mu(\mu-1)(1-\lambda)+2 \lambda) b_{1}=\gamma\left(h_{2}-p_{2}\right),
$$

that is,

$$
\left|b_{1}\right| \leq \frac{|\gamma|\left(\left|h_{2}\right|+\left|p_{2}\right|\right)}{|2(\mu(\mu-1)(1-\lambda)+2 \lambda)|} .
$$

By squaring and adding (16) and (19), using (22) in the computation leads to

$$
b_{1}^{2}=\frac{\gamma^{2}}{(\mu-\lambda-2 \lambda \mu)^{2}}\left(\frac{h_{2}^{2}+p_{2}^{2}}{2}-\frac{[\mu(\mu-1)(1-\lambda)+2 \lambda]^{2}\left[h_{1}^{2}+p_{1}^{2}\right]^{2}}{16(\mu-\lambda \mu-\lambda)^{2}}\right) .
$$

that is,

$$
\left|b_{1}\right| \leq \frac{|\gamma|}{|(\mu-\lambda-2 \lambda \mu)|}\left(\sqrt{\frac{\left|h_{2}\right|^{2}+\left|p_{2}\right|^{2}}{2}+\frac{[\mu(\mu-1)(1-\lambda)+2 \lambda]^{2}\left[h_{1}^{2}+p_{1}^{2}\right]^{2}}{16(\mu-\lambda \mu-\lambda)^{2}}}\right) .
$$

From (26) and (28) we get the desired estimate on the coefficient $\left|b_{1}\right|$ as asserted in (9).

In order to find the estimate $\left|b_{2}\right|$, consider the sum of (17) and (20), we have

$$
b_{0} b_{1}=\frac{\gamma\left(h_{3}+p_{3}\right)}{2 \mu(\mu-1)(1-\lambda)-(1-\lambda) \mu+5 \lambda+4 \lambda \mu} .
$$

Subtracting (20) from (17) with $h_{1}=-p_{1}$, we obtain

$\frac{2(\mu-\lambda-3 \lambda \mu) b_{2}}{\gamma}=h_{3}-p_{3}-\frac{(\mu-\lambda-3 \lambda \mu) b_{0} b_{1}}{\gamma}-\frac{[\mu(\mu-1)(\mu-2)(1-\lambda)-6 \lambda] b_{0}^{3}}{3 \gamma}$.

Using (21) and (30) in (31) give to

$$
\begin{aligned}
b_{2}= & \frac{\gamma}{2(\mu-\lambda-3 \lambda \mu)}\left[\frac{(\mu(\mu-1)(\mu-2)(1-\lambda)-6 \lambda) \gamma^{2} p_{1}^{3}}{3(\mu-\lambda \mu-\lambda)^{3}}\right. \\
& +\frac{2 \mu(\mu-1)(1-\lambda)+8 \mu \lambda-2 \mu+6 \lambda}{2 \mu(\mu-1)(1-\lambda)-(1-\lambda) \mu+5 \lambda+4 \lambda \mu} h_{3}
\end{aligned}
$$




$$
\left.-\frac{2 \mu(\mu-1)(1-\lambda)+2 \mu \lambda+4 \lambda}{2 \mu(\mu-1)(1-\lambda)-(1-\lambda) \mu+5 \lambda+4 \lambda \mu} p_{3}\right]
$$

This evidently completes the proof of Theorem 2.1.

If we take $\lambda=1$ in Theorem 2.1, we get the following Corollary.

Corollary 2.2. Let $g(z)$ be given by (1.2) be in the class $\Sigma_{\lambda, \beta}^{\prime}(\alpha)$. Then

$$
\begin{gathered}
\left|b_{0}\right| \leq \min \left\{\sqrt{\frac{|\gamma|^{2}\left(\left|h_{1}\right|^{2}+\left|p_{1}\right|^{2}\right)}{2}}, \sqrt{\frac{|\gamma|\left(\left|h_{2}\right|+\left|p_{2}\right|\right)}{2}}\right\}, \\
\left|b_{1}\right| \leq \min \left\{\frac{|\gamma|\left(\left|h_{2}\right|+\left|p_{2}\right|\right)}{4}, \frac{|\gamma|}{|\mu+1|}\left(\sqrt{\frac{\left|h_{2}\right|^{2}+\left|p_{2}\right|^{2}}{2}+\frac{\left(\left|h_{1}\right|^{2}+\left|p_{1}\right|^{2}\right)^{2}}{4}}\right)\right\} .
\end{gathered}
$$

and

$$
\left|b_{2}\right| \leq \frac{|\gamma|}{2|(2 \mu+1)|} \times\left[2 \gamma^{2}\left|p_{1}\right|^{3}+\frac{6(\mu+1)}{5+4 \mu}\left|h_{3}\right|+\frac{2(\mu+2)}{5+4 \mu}\left|p_{3}\right|\right] .
$$

If we take

$$
h(z)=p(z)=\left(\frac{1+\frac{1}{z}}{1-\frac{1}{z}}\right)^{\alpha}=1+\frac{2 \alpha}{z}+\frac{2 \alpha^{2}}{z^{2}}+\cdots,(0<\alpha \leq 1, z \in \triangle),
$$

and

$$
h(z)=p(z)=\frac{1+\frac{1-2 \beta}{z}}{1-\frac{1}{z}}=1+\frac{2(1-\mu)}{z}+\frac{2(1-\mu)}{z^{2}},(0<\mu \leq 1, z \in \triangle),
$$

respectively, in the Theorem 2.1, we obtain the following results which is an improvement of estimates obtained by Srivastava et. at $[9$.

Corollary 2.3. Let $g(z)$ be given by (2) be in the class $\Sigma_{\lambda, \beta}^{\prime}(\alpha)$. Then

$$
\left|b_{0}\right| \leq 2 \alpha
$$

and

$$
\left|b_{1}\right| \leq \frac{2 \sqrt{5} \alpha^{2}}{\lambda+1} .
$$

Corollary 2.4. Let $g(z)$ be given by (2) be in the class $\Sigma_{\lambda, \beta}^{\prime}(\mu)$. Then

$$
\left|b_{0}\right| \leq 2(1-\mu)
$$

and

$$
\left|b_{1}\right| \leq \frac{2(1-\mu) \sqrt{4 \mu^{2}-8 \mu+5}}{\lambda+1} .
$$

Remark 2.2. For function $g \in \Sigma_{h, p}^{\prime}(\lambda, \mu)$ given by (2) by taking $p(z)=h(z)=$ $\left.\frac{1+A z}{1+B z}-1 \leq B<A \leq 1\right)$, we obtain the initial coefficient estimates $\left|b_{0}\right|,\left|b_{1}\right|$, and $\left|b_{2}\right|$ which leads to the results discussed in Theorem 2.2 of 15 . 


\section{REFERENCES}

[1] Alamoush, A. G., Darus, M., Faber polynomial Coefficients estimates for a new subclass of meromorphic bi-univalent functions, Advances in Inequalities and Applications, 2016:3 (2016).

[2] Deniz, E., Certain subclasses of bi univalent functions satisfying subordinate conditions, Journal of Classical Analysis, 2(1) (2013), 49-60.

[3] Deniz, E., Yolcu, H. T., Faber polynomial coefficients for meromorphic bi-subordinate functions of complex order, AIMS Mathematics, 5(1) (2020), 640-649.

[4] Deniz, E., Jahangiri, J. M., Kina, S. K., Hamidi, S. G., Faber polynomial coefficients for generalized bi-subordinate functions of complex order, Journal of Mathematical Inequalities, 12(3) (2018), 645-653.

[5] Kapoor, G. P., Mishra, A. K., Coefficients estimates for inverses of starlike functions of positive order, Journal of Mathematical Analysis and Applications, 329(2) ( 2007), 922-934.

[6] Çăglar, M., Deniz, E., Initial coefficients for a subclass of bi-univalent functions defined by Salagean differential operator, Communications Faculty of Sciences University of Ankara Series A1-Mathematics and Statistics, 66(1) (2017), 85-91.

[7] Schober, G., Coefficients of inverses of meromorphic univalent functions, Proceedings of the American Mathematical Society 67(1) (1977), 111-116.

[8] Springer, G., The Coefficients problem for schlicht mappings of the exterior of the unit circle, Transactions of the American Mathematical Society, 70 (1951), 421-450.

[9] Srivastava, H.M., Joshi, B.S., Joshi, S.S., Pawar, H., Coefficient estimates for certain subclasses of meromorphically bi-univalent functions, Palestine Journal of Mathematics, 5 (2016), 250-258.

[10] Babalola, K. O., On $\lambda$-pseudo-starlike functions, Journal of Classical Analysis, 3 (2013), 137-147.

[11] Schiffer, M., On an extremum problem of conformal representation, Bulletin de la Socit Mathmatique de France, 66 (1938), 48-55.

[12] Duren, P. L., Coefficients of meromorphic schlicht functions, Proceedings of the American Mathematical Society, 28 (1971), 169-172.

[13] Hamidi, S. G., Halim, S. A., Jahangiri, J. M., Coefficients estimates for a class of meromorphic bi-univalent functions, Comptes Rendus Mathematique, 351 (2013), 349-352.

[14] Hamidi, S. G., Janani, T., Murugusundaramoorthy, G., Jahangiri, J.M., Coefficient estimates for certain classes of meromorphic bi-univalent functions, Comptes Rendus Mathematique, 352 (2014), 277-282.

[15] Janani, T., Murugusundaramoorthy, G., Vijaya, K., New subclass of pseudo-type meromorphic bi-univalent functions of complex order, Novi Sad Journal of Mathematics, 48(1) (2018), 93-102.

[16] Kubota, Y., Coefficients of meromorphic univalent functions, Kodai Mathematical Seminar Reports, 28(2-3) (1977), 253-261. 